\title{
EDITORIAL AND COMMENT What Will It Take to Reduce the App Gap?
}

\author{
Lyn Paget, $M P H^{7}$ and Dominick L. Frosch, PhD $D^{2,3}$ \\ ${ }^{1}$ Health Policy Partners, Boston, MA, USA; ${ }^{2}$ Palo Alto Medical Foundation Research Institute, Palo Alto, CA, USA; ${ }^{3}$ Department of Medicine, University \\ of California Los Angeles, Los Angeles, CA, USA.
}

J Gen Intern Med 31(12): 1408-9

DOI: $10.1007 / \mathrm{s} 11606-016-3831-\mathrm{y}$

(c) Society of General Internal Medicine 2016

$\mathrm{R}$ ight now you may be reading this editorial on your phone or tablet. Today we often work, play, plan travel, chat, read, bank, dine, or shop with the help of a mobile application (app). Consumers can browse from over 4 million Apple and Android mobile apps in an ever-increasing number of lifestyle categories. ${ }^{1}$ For adult users, the estimated average time spent on mobile digital media has increased from approximately $20 \mathrm{~min}$ per day in 2008 to close to $3 \mathrm{~h}$ per day in 2015 . $^{2}$ For those around to remember, our relationship with mobile technology began in 1973 with the first mobile phone. Weighing in at about 2.5 pounds and costing close to $\$ 4,000$, the early adopters incurred hefty monthly fees and costs for minutes used. Thirty-four years later, Apple introduced the increasingly ubiquitous iPhone. These and other key milestones in the mobile digital media story have shaped an accelerating trajectory of ready access to information, instantaneous communication and unprecedented global connection.

In 2011, $35 \%$ of Americans owned a smartphone. By 2015, that number jumped to $64 \%$ and the global mobile app market hit revenues of $\$ 41.1$ billion. ${ }^{3}$ It is projected that by 2020 , consumers will spend over $\$ 100$ billion on mobile apps. ${ }^{4}$ The rapid growth in mobile apps has captured the imagination of entrepreneurs who believe that they can help solve critical healthcare challenges, particularly in the management of chronic conditions. In 2015, venture capital firms and others invested a total of $\$ 4.5$ billion in digital health products with analysts predicting mobile health tools to be a top health industry investment trend for $2016 .{ }^{5}$ But, will market forces alone be enough to generate high quality products with genuine value across diverse users? With the abundance of mobile health apps currently available, how many are actually making a difference?

Consumers are certainly changing the game, and attitudes are changing too. According to an industry survey, $60 \%$ of consumers report willingness to have video visits with clinicians through a mobile device and $88 \%$ are willing to share personal health data with clinicians to find new treatments. ${ }^{6}$ Other surveys underscore the increasing interest on the side of

Published online August 4, 2016

clinicians, with $46 \%$ indicating they will introduce mobile apps to their practice in the next 5 years; $86 \%$ reporting that health apps will increase their knowledge of their patients' conditions; and $72 \%$ saying health apps will encourage patients to take more responsibility for their health. ${ }^{6}$

These trends tell us that enthusiasm for mobile health apps is high, yet the study by Sarkar and colleagues in this issue of $J G I M^{7}$ highlights critical issues that infuse a dose of reality, especially for investors and app developers, but also health systems considering the use of health apps for enhancing care. The overarching takeaway of this study is what the authors term "suboptimal usability." Although only a modest number of apps were assessed, user experience lacked a variety of key features we have grown accustomed to as we use our phones to pull up relevant information and guide our choices in other arenas. Entering information was a cumbersome and confusing process for most users, and the output of synthesized personal data was difficult to retrieve and often not relevant to specific health or disease management concerns. Testing these basic user needs in a consumer-driven design process with diverse populations is part of the solution and an area where ample and growing knowledge exists. But perhaps more important to achieving the real value of mobile health apps is to also recognize the unique nature of digital support for health-related decisions, and both the potential benefit and risk inherent in their use.

Many other industries can teach us how to support consumers with mobile tools that have user-friendly interfaces and features, yet those tools often guide decisions with less serious implications. Although disappointing, a low quality dinner experience at a restaurant chosen by an app with crowdsourced data can be chalked up to a learning experience. A mismanaged medication dose guided by a mobile health app is very different. Evident in these higher-stakes decisions is the need for these apps to meet a higher standard before they can be viewed as trusted sources of information and receive endorsement from clinical care teams.

Understanding what actually works in the mobile health world is essential if we are to achieve sustained benefit from these products. The sheer number of apps alone is creating an overwhelming abundance of choices without clear guidance on quality and value. The lack of evidence on the benefit of health apps has increased involvement of the Federal Trade 
Commission and prompted reviews of several apps claiming to provide accurate diagnostic and treatment advice. In 2015, several manufacturers were scrutinized, fined and barred from making these deceptive product claims. ${ }^{8}$ These apps ranged from promising improvement in attention deficit/hyperactivity disorder (ADHD) symptoms to detecting and diagnosing melanoma. All were found to have no scientific basis for their marketing claims. Of particular concern are apps that may prey on consumers who rely on them to diagnose and treat conditions as a means to avoid rising medical costs, especially as high deductible health plans become more commonplace. Evaluation frameworks are beginning to be published in the literature; yet just as we are challenged in the health care quality measurement space to develop metrics that are meaningful to patients, we similarly lack a universally accepted method to certify or rate apps that is helpful to consumers. Efforts to bridge this gap have met with little success amidst several expert and multi-stakeholder recommendations for a national approach. ${ }^{9,10}$ While the number of mobile health apps proliferates and consumers continue to embrace their use, the potential for harm escalates. This growing marketplace encouraged by high interest in innovating toward more mobile healthcare needs a complementary force to develop evidence identifying what works and what doesn't.

Beyond the need for more systematic efforts to evaluate mobile health apps, a further unsolved challenge is how to integrate these mobile tools into the larger system of care. Easy access to agile and relevant personal health data has the potential to be a powerful instrument in tailoring care plans that respect individual goals, preferences and limitations. The opportunity for this enhanced care plan increases exponentially when the right data get to the right person at the right time in the right format. This could be patients, clinicians, family members, caregivers or health plans, but it requires interoperability with electronic health records (EHRs) and prioritizing actionable data without demanding excessive cognitive effort from already overburdened clinicians. The promise of mobile that investors admire includes opportunities to streamline care processes, curb costs and leverage technology to deliver more appropriate care. For this vision to evolve, integration is a key part of building the fabric to more efficiently meet the needs of all stakeholders.

It's hard to imagine that the priorities of individuals interested in using mobile health apps would differ greatly from those of clinicians and purchasers. Self managing health and chronic conditions, avoiding unnecessary care, receiving timely decision support and treatment, and reducing excessive costs are important goals that ought to serve as cornerstones for funding and developing mobile health apps. These common goals will need to chart the course to enable apps and digital tools to track and measure data that create profiles to help us understand the drivers of health and disease, as well as the potential impact of interventions. Getting there will require a more systematic look at ways to identify good apps and harmonize across the broad assortment of options to provide consumers with choice and individualized fit. It will also require thoughtful development of an environment that safely provides universal mobile digital support. With evaluation standards in place, clinicians can guide and endorse patient choices while helping to incorporate mobile apps into goal setting and care plans. Purchasers have a large stake in this game and their involvement could be instrumental in catalyzing market forces to either drive quality or simply reinforce the status quo.

In the time it took to read this editorial, close to 20 new apps were submitted to the Apple iTunes store. ${ }^{11}$ We have entered an era that promises to change the way we manage our health and how we interact with the health care system. This transition to digitally supported health could shape an unprecedented approach to managing the multi-dimensional nature of health and disease. As we navigate this new terrain, there is an urgent imperative to engage immediately to shape the future of mobile health for safety, value and optimal benefit. For funders and investors, developers and users, a clearly defined pathway to high quality will direct the resources and produce results that can truly make a difference. If not now, when?

Corresponding Author: Lyn Paget, MPH; Health Policy Partners, Boston, MA, USA (e-mail: lpaget@healthpolicypartners.com).

\section{REFERENCES}

1. Statista. 2016 Number of apps available in leading app stores. Available at: http://www.statista.com/statistics/276623/number-of-apps-availablein-leading-app-stores/.

2. Smartinsights. 2016 Insights from KPCB US and global Internet trends 2015 report. Available at: http://www.smartinsights.com/internet-marketing-statistics/insights-from-kpcb-us-and-global-internet-trends-2015-report/.

3. Pew Research Center. 2015 US smartphone use in 2015. Available at: http://www.pewinternet.org/2015/04/01/us-smartphone-use-in-2015/.

4. Statista. 2016 Worldwide mobile app revenues 2020. Available at: http://www. statista.com/statistics/269025/worldwide-mobile-app-revenue-forecast/.

5. Rock Health. 2016 Digital health funding: 2015 year in review. Available at: https://rockhealth.com/reports/digital-health-funding-2015-year-in-review/.

6. Research Now. 2016 Are mobile medical apps good for our health? Available at: https://www.researchnow.com/mobile-medical-apps-good-health-newstudy-research-now-reveals-doctors-patients-say-yes-infographic/.

7. Sarkar U, et al. Usability of commercially available mobile applications for diverse patients. J Gen Intern Med. 2016. doi:10.1007/s11606-016-3771-6.

8. Morgan Lewis. 2016 Increased FTC enforcement activities as FDA deregulates low-risk health IT devices. Available at: https://www. morganlewis.com/pubs/increased-ftc-enforcement-activities-as-fda-deregulates-low-risk-health-it-devices.

9. National Committee for Quality Assurance. 2014 Building a strategy to leverage health information. Available at: http://www.ncqa.org/hedisquality-measurement/research/building-a-strategy-to-leverage-healthinformation.

10. Powell AC, Landman AB, Bates DW. In search of a few good apps. JAMA. 2014; 18:1851-1852.

11. Statista. 2016 Number of new apps/games submitted to the iTunes store per month. Available at: http://www.statista.com/statistics/258160/ number-of-new-apps-submitted-to-the-itunes-store-per-month/. 